the lungs they are the origin of infarcts and account for the attacks of hæmoptysis which these patients are so subject to. This patient has had several. The bleeding from the lungs is of course influenced by the increased blood pressure, the result of hypertrophy of the right ventricle. The emboli which find a lodgment in the systemic arteries are either pieces of vegetations from the mitral valve or broken up blood clot, usually from the left auricular appendix. These emboli not infrequently fix themselves in the cerebral arteries, usually in those of the left cerebral hemisphere, as being a more direct course from the aorta, and so explain the symptom which we call hemiplegia. So far this patient has escaped this form of paralysis. Embolism may sometimes be suspected in the renal vessels by the patient passing blood in the urine. In mitral stenosis the urine is usually scanty, as the renal vessels are inadequately supplied with blood and the arterial pressure is low.

Dropsy does not occur so often here as in cases of mitral insufficiency and it is not unusual when it shows itself to begin as ascites. The liver is often enlarged and tender and sometimes extreme pain is complained of in this region which depends upon stretching of the liver capsule. The deficiency of the circulation in the stomach and the bowels may give rise to indigestion and even to hæmorrhage.

The impsrfect circulation through the brain would account for the sleeplessness which these patients frequently complain of. Bronchial catarrh is very easily set up on account of the abnormal moisture in the lungs. If the hepatic veins are much dilated a pulsating liver may be present. The blood tends to accumulate on the right side of the heart, notwithstanding Sansom's assertion that the circular muscular fibre in the pulmonary veins exert a powerful sphincter action, which, he says, can be demonstrated, and that it assists in keeping the backward blood pressure away from the lungs in cases both of mitral stenosis and mitral regurgitation.

In giving a prognosis a most favourable sign is a well-marked second sound heard at the pulmonary area, which is sometimes reduplicated. The closure of these cusps is sometimes so forcible that they can be felt by the hand in the second left interspace as a shock. This means that the hypertrophy of the right ventricle is well maintained and in association with this there is a pronounced pulsation in the epigastrium.

In treating these patients rest in bed is often necessary they should at any rate be cautioned not to over-exert themselves. If the venous system is very full of blood opening a vein in the arm is sometimes a good palliative. In this condition of an over-distended venous system no drug is more valuable than blue pill, given perhaps in conjunction with enough compound colocynth pill to produce a watery evacuation. Compound jalap powder is useful for dropsical complications. When the pulse becomes very weak and frequent cardiac tonics are indicated and strychnia or digitalis should be given. If the urine is very deficient the best cardiac tonic to give would be caffeine which is stronger and better than the citrate of caffeine. Insomnia is frequently a troublesome symptom in these cases and it may be necessary to prescribe chloralamid occasionally at bedtime. Easily assimilated, light nourishment is all important, but see that all the food you give is digested, for although sudden death is a very rare occurrence in cases of mitral stenosis it may be induced if the stomach is distended and so allowed to press upon the right heart. I think the cardiac pain is best relieved by advising very little fluid to be taken. A little alcohol is usually required in treating these cases.

The Maida Vale Hospital and Home for INCURABLE CHILDREN. - The President of this institntion, H.R.H. the Dake of Connanght, occupied the chair on the evening of Nov. 25th at the festival dinner in aid of the charity in the Prince's Restaurant, Piccadilly, W. They had met, said His Royal Highness, in the interests of incurable children of the poor who by reason of the nature of their infirmity could not be received in the large hospitals and could not be properly looked after in their humble homes. There were 30 children in the home-15 boys and 15 girls, but more room, better air, and greater nursing facilities were needed and it was proposed to remove to a superior home in South Hampstead. The cost of the transfer would exceed $£ 12,000$, towards which $£ 6000$ had already been subscribed, and for the other $£ 6000$ he appealed to the company present. It was afterwards announced amid cheers that the sum of $£ \$ 400$ had been subscribed at the dinner.

\section{ON THE PRESENT TREATMENT OF THE ENLARGED PROSTATE. ${ }^{1}$}

By C. MANSELL MOULLIN, M.D. Oxon., F.R.C.S. ENG. SENYOR SURGEON TO, AND IECTURER ON SURGERY AT, THE LONDON HOSPITAL; EXAMINER IN SURGERY AT THE UNIVERSITY OF CAMBRIDGE.

THE duty of the person intrusted with introducing a discussion upon a clinical subject such as the treatment of enlargement of the prostate is not so much to read an exhaustive paper on it himself as to define those conditions of the affection which appear to him to be most likely to repay discussion, and to indicate the methods of treatment which, in his experience, are best suited to those conditions, and the reasons for selecting them. In accordance with this idea I wish to leave altogether upon one side all those cases in which catheter life is so successful that the patient manages to lead a comfortable existence by passing a catheter for himself once, or at the utmost twice, in the 24 hours. There is notbing more to be said about them any more than there is about those happy old men who are not even aware that they possess a prostate. The treatment cannot be called ideal. It merely concerns itself with one of the consequences of the enlargement and leaves the cause untouched. But if all the symptoms, both those that are present at the time and those that are likely to occur as the patient grows older, are adequately met by such a simple proceeding, and if the frequency with which the catheter has to be used does not increase as years pass by, it must be allowed that the treatment meets the necessities of the case sufficiently well. Such cases may be placed once for all upon one side. I have nothing further to say about them. I am only con. cerned with the exceptions, those in which this line of treatment is either a failure from the first or is morally certain to become a failure if it is persisted in; and I am sorry to say that it is my conviction that these exceptions are far more numerous than is generally believed. It is so easy to make use of a catheter after the first few attempts and it appears to be so safe, and often it gives such complete relief for a time, that it is difficult for the surgeon, and impossible for the patient, to realise the evils which may, and in a large proportion of instances do, follow its habitual use. If however, the results of ordinary catheter life are carefully recorded and the progress noted year by year there is not the least doubt that in the vast majority there is a steady change for the worse ; and if candid inquiry is made it is impossible to avoid the conclusion that the downward progress is to a very large extent the direct result of the treatment. Enlargement of the prostate is a progressive disease, though it is curious how very seldom this is realised. Neither the patient nor the gland ever remains stationary. The one steadily grows older and feebler, less and less able to help himself, and the other is always increasing in size. The difficulty grows greater and greater as the ability of the patient to deal with it grows less. Year by year the catheter has to be passed more frequently. At last its use becomes almost incessant day and night. The intervals grow shorter and shorter. The pain becomes almost intolerable. The introduction of the instrument becomes more and more difficult until it may be impossible for the patient and even the surgeon may not find it easy. The bladder entirely loses its power. The habitual passage of a catheter is enough to destroy the contractile power of the bladder within two years. Hæmorrhage becomes more frequent. And far worse than any of these, septic inflammation, due to septic organisms carried in by the catheter, is certain to set in at last. Suppurative cystitis, ascending pyelonephritis, phosphatic calculi, septic prostatitis, thrombosis of the prostatic plexus, periprostatic abscess, epididymitis, and urethritis are some of the commonest consequences of the habitual use of catheters in cases of enlargement of the prostate and are the immediate cause of death sooner or later in the majority of cases that are treated by habitual catheterisation.

It is quite true that these septic troubles ought not to

1 The opening paper at the discussion at the meeting of the Harreian Society on Dec. 3rd, 1903. 
occur and that now that is an accepted fact that inflammation of the bladder is always the result of the introduction of septic organisms (except in the case of specific diseases such as typhoid fever), cystitis is not quite so frequent or so severe as it used to be, but it is absolutely impossible for an old man, growing year by year more feeble and less able to take care of himself, who has to pass an instrument upon himself many times by day and night often at a most urgent call, always, on every occasion, to take adequate precautions. Given abundance of time and assistance the greatest skill will scarcely suffice. With few exceptions the habitual employment of catheters means atony of the bladder and septic cystitis sooner or later; and septic cystitis, with enlargement of the prostate, means at last death by slow torture.

These are the cases the treatment of which I propose that we should discuss to-day. In actual practice there is no difficulty in finding out which they are; or if there should be a little difficalty in distinguishing them just at first a few weeks' treatment will always show. In case of doubt the catheter should be given a trial first, but if the frequency with which it has to be used increases; if the main feature of the complaint is not so much vesical inability or mechanical obstruction as irritability of the bladder; if the amount of residual urine grows larger; if there is a tendency to cystitis (for undoubtedly patients differ greatly in the degree of their susceptibility to septic organisms); if attacks of congestion are of frequent occurrence ; if there is a valve-like outgrowth at the orifice of the blaxder or a conspicuous thickening in the posterior median wall of the urethra; if there is any difficulty in the passage of the catheter; if the introduction of the instrument is liable to cause bleeding; if there is a tendency to the formation of calculi in the post-prostatic pouch or if the specific gravity of the urine steadily falls, it is clear that the use of the catheter does not fulfil the necessary conditions and the attempt will end in failure. It may answer for the moment. In the future it certainly will not and the sooner this is recognised and some other method of treatment adopted the better for the patient.

The first essential in the treatment of enlargement of the prostate is to form an accurate opinion as to its nature. It is not sufficient to say merely that the prostate is enlarged or even to describe its shape or size. The cause of the enlargement must be ascertained, whether it is simple congestion of the venous plexuses in and around the neck of the bladder, or actual overgrowth, or the two together in varying proportion. What relation these bear to each other, whether the overgrowth is not, in part at least, the consequence of the prolonged congestion, or whether both are not due to some more remote cause, such as the reflex influence of the testes, is a question of great interest but does not concern us now. We have to deal with the two conditions themselves, usually in combination, and the consequences to which they give rise.

Congestion of the tissues at the neck of the bladder is a much more potent factor (especially in the early days of enlargement of the prostate) in causing the symptoms of which the patient complains than is usually believed. It is the main cause of the early irritability. It greatly increases the susceptibility to inflammation. It weakens the strength of the muscular coat of the bladder by interfering with its blood-supply and at the same time it throws extra work upon the bladder by making it contract more frequently against greater resistance. It may be simply and entirely passive, the result of venous obstruction; or it may be due to the irritation caused by septic organisms or bv an excess of uric acid or oxalates in the urine ; or it may be reflex, the result of stimuli that originate in connexion with the sexual organs. Not uncommonly all these canses are at worit ogether. Its frequency and the freguency with which it ieads to thrombosis of the prostatic plexus may be estimated from the frequency with which hæmorrhage occurs, from the chains of phleboliths which are so rarely absent from the prostatic plexus of old men, and, more than anything else, from the benefit derived in these cases from what is called general or constitutional treatment. I have not to deal to-day with this : I am only concerned with local measures, but I cannot help pointing out that the undoubted value of constitutional treatment depends entirely upon the effect that it has upon this congestion, and in every case in which congestion at the neck of the bladder is a prominent feature constitutional measures, such as rest in bed, hot baths, dieting, and free purgation, must never be omitted. Alone, when the congestion is chiefly passive, they are often sufficient of themselves to give relief, and though unfortunately they are not so beneficial when the congestion depends upon other conditions they are still of great value.

The local treatment of enlargement due to congestion need not detain us long. As a rule local interference with a congested organ tends to make the congestion worse. But there is one method which is not popular because $I$ believe it is not well known but which I have found of great benefit. I allude to Bottini's cautery as modified by Freudenberg. The proceeding is very simple. The bladder is distended with air so as to keep the rest of the wall out of danger and three parallel incisions are made with the white-hot blade of the cautery, one along the floor in the middle line of the neck of the bladder, the others one on each side. An anæsthetic is not necessary if the patient is accustomed to instruments though I think it is advisable. There is very little pain afterwards and $I$ have not met with any case in which the hæmorrhage was worth mertioning. The venous plexuses in the submucous and mucons layers and the nerves, which in this region are so exceedingly sensitive, are divided; and three linear cicatrices are left which contract and distinctly lessen the tendency to subsequent congestion. I cannot recommend this method for enlargement due wholly or even in great measure to overgrowth of the gland. It is inefficient for this and there are other better methods. But in cases in which there are but little growth and much congestion, and in which there is great irritability of the bladder with the imperative necessity for immediate micturition as a prominent feature, and in which the muscular coat of the bladder is beginning to fail, a respite can certainly be obtained, if not a cure, without serious risk. Whether it has any effect in checking the growth I should not like to say but $I$ do not consider that it is probable.

The only other local measure that needs consideration in dealing with prostatic congestion is division of the vasa deferentia or, rather, of the nerves that accompany and surround them. There is no doubt that this operation (and, of course, the other operations that entail the same results upon the nerves coming from the testes) does check to some extent the liability of the prostate to congestive attacks and, so far as these attacks are dependent upon stimuli originating from the testes, it is of some benefit, while it certainly does not expose the patient to any serious degree of risk. As, however, division of the vasa deferentia cannot have any effect upon passive congestion, or upon that which is caused by septic organisms or by irritating substances in the urine, the operation is very often a failure.

The other form of enlargement of the prostate, that which is due to irregular glandular overgrowth, admits only of mechanical treatment. It may lead to obstruction, so that no urine can be passed voluntarily, or it may cause intense irritability of the bladder, according to the direction in which it grows and the amount of congestion that attends it, but whatever it does if palliative measures, such as treatment by catheters, fail, and they nearly always do sooner or later, there is no alternative but removal in some form or other. Drainage can only be regarded as the last resource when the patient is too far gone to stand anything else.

Removal can be effected either by inducing atrophy by means of some operation upon the testes or the spermatic cords or by operating upon the enlargement itself. Of these two methods, the former, like everything else which is novel, was boomed too much and now has met the usual fate by falling into, I cannot help thinking, somewhat undeserved discredit. That it benefited a very large proportion of casesa proportion, according to American surgeons, as high as 85 per cent.- there can be no doubt; and equally there can be no doubt that it failed sometimes (especially in those cases in which the enlargement was largely due to inflammation) and that it was attended by a much higher rate of mortality than would have been expected from the severity of the operation. Moreover, certain cases were recorded in which very grave mental disturbance, imbecility and even mania, followed the operation; it is even stated that insanity followed the division of one of the vasa deferentia under cocaine, and though I do not believe for a moment myself that the brain and testes in old men are in such close functional relationship with each other as this would imply, the fear of such an occurrence, the strong sentimental objection to the operation, and the success attending removal of the enlargement by operation, all combined, have caused this procedure to fall completely into the background.

An enlarged prostate can be removed more or less com pletely either through a perineal or a suprapubic opening. 
When the bladder is very small and rigid from repeated attacks of cystitis and the prostate is very hard and dense the former method is indicated. Under all o'her conditions the prostate, as from the nature of its surroundings it must grow up towards and into the bladder, is best approached through the bladder. 14 years ago McGill advocated and performed this operation and though various modifications have been introduced since then it remains in all essential details the same. McGill described how the growth was to be enucleated with the finger after dividing the mucous membrane of the bladder and himself enucleated and exhibited both lateral lobes removed in this way. In $1892,{ }^{2}$ in the Hunterian Lectures delivered before the Royal College of Surgeons of England, I pointed out that " if the operation is to prove successful it resolves itself into removing the whole of the vesical mass, whether it springs from the lateral lobes, or is an upgrowth from the posterior wall, or is a detached nodule, and then extending the exploration down the whole length of the prostatic urethra," and I cited some instances in which the operation had proved, or nearly proved, a failure because the growth had not been followed down the prostaic urethra. That was the operation I performed then, following McGill's directions; it is the operation that I have continued to perform since, it is the operation that is performed now generally, and it is one that I can thoroughly recommend for these cases. A certain amount of confusion, it is true, has been caused by erroneous description of what has been called the capsule of the gland. But it has been conclusively shown by Shattock and Wallace that the covering on the enucleated masses is a pathological product formed around those masses out of the surrrounding tissues by the way in which they press upon them and condense them in their growth. The larger and the more lobular the masses of which the enlarged prostate is composed the more easily can they be shelled out, because of this fictitious capsule which they form around them; and there can be no doubt that for this form of enlargement this method of operating will not only supersere all others but will do away with the treatment by habitual catheterisation for which these cases are by no means suited. Only if the prostate in addition to being enlarged from glandular over growth is in a state of intenre congestion, this must be allowed $t$ in subside first under the influence of the constitutional treatment to which I have already alluded, and the patient must be warned that if the musoular coat of the bladder has been ruined already by catheterisation and cystitis, removal of the enlarged prostate cannot restore it, thongh it will help it by rendering its work more easy.

Wimpole-street, W.

\section{A CASE OF CHRONIC INTUSSUSCEPTION; EXCISION OF 42 INCHES OF SMALL INTES IINE ; RECOVERY.}

\section{BY F. C. Wallis, B A., M B., B.C. Cantab.,} F.R C.S. ENG,

assistant surgeon to chaRING Cross hospital; SURgeON to The METROPOLITAN HOSPITAL.

A HEALTHY looking married woman, aged 32 years, was admitted into Charing Cross Hospital on April 14th, 1903, suffering from intestinal colic. She was in perfect health until May, 1901. Previously to this she had never had a day's illness in her life The bowels always acted with great regularity and aperients were rarely necessary She had never suffered from diarrhœa. The present illness began in May, 19:1, with a sudden onset of most acute pain in the abdomen (there was no cause for it as far as she knew). The pain was of a colıcky character but was not sufficiently bad to make her take to bed until the end of the week when the attacks became more frequent and vomiting commenced. Food always excited the colic and romiting always followed it. She described an attack as a "general turning round-and-round feel in the stomach, followed quickly by vomiting." This attack lasted for

2 The Lancer, June 4th (p. 1229), 11th (p. 1287), and 18th (p. 1354), 1892 A paper read before the Clinical Society_of London on Nov. 27th, three weeks and she was in Guy's Hospital for the latter part of it. Atter this she was perfectiy well until August, 1901, when she had a similar attack and there was a third attack in September, 1901. These attacks resembled the first except that the bowels were obstinately constipated for three or four days. When the bowels acted she was much relieved and the attack would generally pass off. There was no constipation at the beginning of an attack but pain and vomiting preceded it for three or four davs. After the third attack she continued well until June, 1902, when there was another attack, since when there had been many milder in degree but all similar in character to the first. She had never passed any blood or mucus in the motions. The abdomen never became swollen and pressure neither inereased nor relieved the pain. No tumour had been felt. She was admitted into the medical ward of Charing Cross Hospital during one of these attacks of pain and was in for five days, leaving on April 18th, 1903, apparently quite well. She was re-admitted on April 22nd for another attack of pain, during which vomiting was more or less continuous; it was bilestained but not offensive. The abdomen was lax and not tender (this condition existed during all these attacks). On the $25 \mathrm{th}$ an indefinite mass was felt below and to the left of the umbilicus.

It was decided that unless a distinct improvement took place in the next 24 hours an exploratory laparotomy should be performed. As the patient was no better-in fact, getting weak-I operated at 11 A M. on the $25 \mathrm{~h}$. When the patient was under the anæsthetic a large freely moveable mass could be felt in the left umbilical region. The abdomen was opened in the mid-line beluw the umb licus by an incision which was lengthened to five inches in all. The palpable mass was an intussusception involving small intestine only. The length of bowel involved amounted to three feet six inches. The proximal gut was materially dist' nded. An unsuccessful attempt was made to reduce the invagination and enterectomy was decided upon. Whilst lifting the involved gut out of the abdomen the distal end tore like a piece of wet blotting-paper close to the end of the intus-usception and it was noticed that this part of the buwel was much inflamed. The intestinal contents were expressed at either end and Lane's intestinal clamps were applied on healthy bowel. The bowel was divided first at the upper end and the mesentery was cut through parallel to the intestine, the ressels being clamped as they were cut. The intestine was divided at the lower end and the two ends were united with a Murphy's button. A few Lembert sutures were put in in addition and the cut edges of the mesentery were sewn together with silk sulures, the vessels being ligatured with silk also. Some hæmorrbage found its way between the layers of the mesentery. The parietal wound was closed by one row of separate fishinggut sutures. The operation lasted for one hour. The patient stood it very well and her pulse was 120 at the end. One pint of beef-tea was given by the rectum. There was practically no shock from the operation.

The patient was nourished by rectal enemata only for two days ; on the third day small quantities of plasmon and thick barley-water were given by the mouth and on this day the bowels acted twice, the motions being well formed. The food by the mouth was gradually increased and the rectal feeding was discontinued, the stitches were removed on the eighth day, and everything went very well indeed until May $15^{\circ} \mathrm{h}$ (three weeks after the operation) when the patient had an attack of pain similar to those she had previously suffered from: paroxysmal colic with intervals free from pain. Chloroform was administered and on palpating the abdomen I could feel the button in what I thonght was the de:cending colon. It was quite easily pushed down into the pelvis but it could not be reached by the rectum. No button was passed and the pains continued. On May 18th the button was seen by the $x$ rays to lie on the outer side of the right rectus, two inches above the inlet of the relvis. On the 19th I reopened the abdomen and found the button about four inches above the ileo cæcal valve; it could not be passed on into the colon so I removed the button throngh a longitudinal incision which was sewn up transversely. From this second operation the patient made a perfect recovery and left the hospital quite well on June 10th. I saw her recently and she was in the best of health.

The following is the pathologist's description of the snecimen: "No. 1223. Enteric intussusception in adult. 42 inches of small gut excised. A portion of small intestine, 67 centimetres ( $26 \frac{1}{2}$ inches) of which forms the outer 